\title{
APUNTES SOBRE EL ESTADO DEL ARTE DE LA EXPERIENCIA CORPORAL ${ }^{1}$
}

\author{
NOTES ON THE STATE OF THE ART OF CORPOREAL EXPERIENCE
}

\author{
Libardo Mosquera Mateus ${ }^{2}$ \\ Sonia López Domínguez ${ }^{3}$ \\ Martha Arenas Molina ${ }^{4}$
}

\section{Resumen}

El presente artículo recoge investigaciones, estudios y artículos desarrollados alrededor del término experiencia corporal, expresión de complejo abordaje para su análisis, por cuanto es reciente el trabajo que se ha dado a su alrededor, en especial en el aspecto educativo. Este artículo presenta un recorrido por algunos autores que han posibilitado el estudio del mismo desde lo filosófico y pasando por el trabajo realizado desde otras disciplinas y la educación. Pretende aportar en algunas concepciones acerca de la experiencia corporal, tema que ocupa e interesa por ser el objeto de estudio de la Licenciatura en Educación Física de la Universidad Pedagógica Nacional.

Palabras claves: experiencia; experiencia corporal; educación corporal

\section{Abstract}

This article gathers research studies and papers developed regarding the term corporeal experience; which is both complex and new, in terms of its analysis, because it has only been recently an object of study in the education field. This paper offers an overview of the contributions of some authors in relation to its definition, from the philosophical and educational perspectives, as well as from other disciplines. It aims to provide some key ideas about the corporeal experience, topic that is being studied at the Universidad Pedagógica Nacional's Bachelor degree program in Physical Education.

Keywords: experience; corporeal experience; corporeal education

Fecha de recepción: 23 de octubre de 2015

Fecha de aprobación: 8 de marzo de 2016

Para citar este artículo:

Mosquera, L., López, S. y Arenas M. (2016). Apuntes sobre el estado del arte de la experiencia corporal. Lúdica Pedagógica, (23), 119-126.

1 Resultados del proyecto de investigación: Representaciones Sociales sobre el concepto de experiencia corporal, que tienen los estudiantes de los semestres de sistematización del PCLEF, financiado por el Centro de Investigaciones de la Universidad Pedagógica Nacional. Código FEF 329-14.

2 Magíster en Sociología de la Educación, UPN; especialista en Pedagogía y Didáctica de la Educación Física, UPN; licenciado en Educación Física, UPN. Doctorando en Educación de la Universidad Internacional Iberoamericana de México. Docente catedrático, Facultad de Educación Física, Universidad Pedagógica Nacional. Correo electrónico: Imosquera@pedagogica.edu.co

3 Magíster en Educación y Desarrollo Social, CINDE-UPN; especialista en Investigación sobre el Fenómeno de las Drogas, Universidad de Sao Paulo; especialista en Docencia Universitaria Universidad. El Bosque; licenciada en Educación Física, UPN. Docente de planta, Facultad de Educación Física, Universidad Pedagógica Nacional. Correo electrónico: solopez@pedagogica.edu.co

4 Magíster en Educación, Pontificia Universidad Javeriana; especialista en Gerencia Educativa, Universidad de la Sabana; especialista en Pedagogía y Docencia Universitaria, Universidad Gran Colombia; licenciada en Lingüística y Literatura, Universidad Distrital. Docente ocasional de tiempo completo, Facultad de Educación Física, Universidad Pedagógica Nacional. Correo electrónico: marenas@pedagogica.edu.co 


\section{INTRODUCCIÓN}

Dentro de los estudios e investigaciones sobre la experiencia corporal, existen diversos significados del concepto que hacen complejo su abordaje, en particular cuando los dos vocablos tienen variadas interpretaciones. Si se habla solo de la palabra experiencia, para la filosofía se trata de un término que designa hechos de naturaleza diversa. Como lo señala (Amengual, 2006), para los empiristas existe una relación directa entre experiencia y conocimiento. Su visión es un asunto gnoseológico:

Experiencia es, pues, de entrada ese algo, lo otro respecto del entendimiento, en relación con lo cual se puede y se debe plantear la cuestión de la certeza como objetividad. Así se presentan, en términos generales, las filosofías de la experiencia o empiristas. (Amengual, 2006, p. 6).

El mismo autor en su texto analiza las transformaciones que tuvo el concepto de experiencia en Kant y Hegel. Señala que para Kant la experiencia es algo más que razón "la unificación de lo múltiple de las sensaciones no es algo posterior a la experiencia, sino lo que la constituye, constituyendo al mismo tiempo el conocimiento" (Amegual, 2006, p. 12).

En la introducción de la fenomenología del espíritu, Hegel define la experiencia como "este movimiento dialéctico que la conciencia lleva a cabo en sí misma, tanto en su saber cómo en su objeto, en cuanto surge ante ella el nuevo objeto verdadero, es propiamente lo que se llamaría Experiencia" (Hegel, 1995, p. 58). Ese objeto nuevo, como lo señala Gama, está relacionado con un término de la negación utilizado por Hegel y que se interpreta como

Uno tiene experiencias, en el sentido de un saber obtenido a través de una larga praxis: pero uno hace experiencias cuando algo nuevo defrauda nuestras opiniones típicas. A través de esta decepción uno aprende que las cosas "no se habían visto correctamente hasta ahora" y en consecuencia se produce un mejor saber de éstas. Esta negatividad de la decepción constituye así el momento esencia del movimiento de la experiencia. (Gama, 2002, p. 55).

Si en Hegel se señaló el concepto de negatividad para darle sentido a nuevas experiencias, en Gadamer, que se distancia de la comprensión de la experiencia a partir de los resultados científicos, tiene valor el movimiento de la conciencia, que da posibilidad o apertura a nuevas experiencias. Al respecto, Gama en el análisis que hace del concepto de movimiento de la experiencia define: que el movimiento de la experiencia es un movimiento dialéctico, y esto significa que aquel que hace una experiencia avanza por un camino a lo largo del cual se apropia de un saber siempre es más rico y abarcante, un saber que no anula las opiniones que previamente fueron tornadas por verdaderas, sino que les otorga su lugar correcto al interior de una nueva verdad más amplia. Todo esto tiene lugar en tanto que la conciencia es su experiencia poniéndola a prueba permanentemente su "sistema de opiniones y prejuicios se invierte sobre sí" (Gama, 2002, p. 58).

Con esta sucinta interpretación de algunos filósofos sobre la experiencia, se evidencia que abordar el concepto de experiencia es una tarea compleja, se le puede relacionar con el conocimiento, con la historia de vida que cada uno va construyendo, pero también con las subjetividades que hacen algo particular y significativo para cada persona. Las múltiples interpretaciones de la experiencia hacen que sea en la actualidad un tema de análisis para diversas disciplinas. La revisión que se hizo sobre el estado de arte de la experiencia corporal así lo evidenció; hay estudios sobre la experiencia corporal desde la sociología, la psicología, las artes y la educación, entre otros; es "un tema de debate en la filosofía contemporánea aunque se aborde en otros términos, como sí-mismo, subjetividad, existencia, identidad personal, identidad narrativa, etc." (Amengual, 2006, p. 6).

Ahora bien, si nos referimos no solo al término experiencia, sino que lo adjetivamos con lo corporal, se infiere que desde este sentido toda experiencia se configura en lo corporal, se comprende como un acto trascendente, que es irrepetible y que da sentido a nuestra existencia. En esta visión se concibe lo corporal como la conjunción dinámica y reflexiva de la naturaleza humana con el entorno cultural, que constituye nuestra corporeidad, que es el mismo cuerpo vivido, una experiencia como ser en el mundo. Desde esta comprensión de la experiencia, el concepto de cuerpo debe superar el dualismo antropológico que lo ha interpretado como materia, distintivo y separado del alma y entenderlo como corporeidad, que puede ser traducido a partir del 
vocablo alemán Leib, ${ }^{5}$ que en palabras de Planella "nos sitúa en la órbita de lo subjetivo, sensible, vivencial y experimental (frente a lo exclusivamente objetivado y materializado). La corporeidad es la lectura del cuerpo desde su dimensión simbólica" (Planella, 2006, p. 47) y que Duch la define así:

Cuando afirmamos que el cuerpo humano es corporeidad queremos señalar que es alguien que posee consciencia de su propia vivacidad, de su presencia aquí y ahora, de su procedencia del pasado y de su orientación al futuro, de sus anhelos de indefinido a pesar de su congénita finitud. (Duch, 2003, p. 282, citado por Planella, 2006, p. 47).

Al hablar de corporeidad, se comprende el cuerpo como unidad, porque desde allí sucede la experiencia corporal como un acontecer humano trascendente y con sentido, que es susceptible de ser investigada o estudiada. Incluso en disciplinas que privilegian el estudio del cuerpo desde lo biológico, como por ejemplo, la medicina, en donde se concibe el cuerpo como objeto, y tiene sentido su conocimiento, funcionamiento y acciones que reconstruyen sus órganos que lo componen; pero desde esta ciencia, también se puede entender el cuerpo desde lo subjetivo. Como lo señala el doctor López-Ibor Juan J.:

Existen dos perspectivas sobre el cuerpo humano en medicina: el cuerpo como objeto y el cuerpo como sujeto. La primera trata del cuerpo de la anatomía y fisiología y en él interesa investigar síntomas y signos, recuperar estructuras y funciones y reducir la discapacidad. La segunda se interesa por la experiencia del propio cuerpo, unas veces porque está afectada la representación global del cuerpo, como sucede en algunas enfermedades del sistema nervioso central, otras porque hay una alteración de la experiencia del cuerpo viviente en el mundo. A esta última llamamos corporalidad. (s.f.).

5 Según Planella (2006, p. 47): “La diferenciación entre Körper y Leib es una precisión terminológica y conceptual compartida por buena parte de los fenomenólogos"; además, Planella señala el aporte hecho por Edith Stein, "cuerpo (Leib) está caracterizado y diferenciado de carne (Körper) pura y materialmente que lo constituye, a través de todos sus estados y sus accidentes" (Stein, 1992; p. 57, citado por Planella, 2006, p. 47).

Según Eichberg (1995, citado por Gallo, 2010, p. 53): "La fenomenología prefirió Leib a Körper. Se describió a Leib como el cuerpo viviente, vivo, avivado y animado, como el cuerpo vivido desde el interior Leib fue considerado como algo más que Körper. Mientras Körper es 'sólo' el cuerpo, Leib es algo más alto, profundo, rico, algo más humano. Leib es el cuerpo más el alma".
Desde esta visión, que entiende que toda experiencia se configura en lo corporal se hizo la revisión de los estudios e investigaciones y se clasificaron en dos temáticas: la primera la experiencia como vivencia y la segunda llamada la experiencia en la educación corporal.

\section{LA EXPERIENCIA Y LO CORPORAL}

Si hablamos de la experiencia sin relacionarla con el conocer, "el objetivo de la ciencia es objetivar la experiencia hasta que quede libre de cualquier momento histórico" (Gama, 2002, p. 51), se podría señalar que al referirnos a la experiencia la relacionamos con lo corporal; toda experiencia acontece y toma sentido en el cuerpo.

Desde este enfoque, la experiencia sucede en la corporeidad que involucra los sentimientos, sensaciones y afectos. A partir de esta perspectiva, en la revisión de las investigaciones y estudios sobre la experiencia, se encontraron algunas líneas o enfoques; una de ellas es la experiencia como una vivencia de vida. En general, este tipo de estudios o investigaciones están caracterizados por las experiencias que tienen determinados grupos poblacionales en situaciones de adversidad ya sea de tipo social o físico. Sin desconocer que son muchos los estudios que presentan esta visión, se señalan solo algunos, que sirven como referentes y además son abordados desde ciencias como la sociología y la medicina que sin ser disciplinas de corte específico educativo son pertinentes para la comprensión del significado de experiencia corporal.

Una de ellas es la de Soru y Duero (2011) llamada "Experiencias corporales y sentimiento de orientación vital: una aproximación fenomenológica y narrativa". En esta investigación, como señalan sus autores, se "analizan las experiencias corporales y las variaciones en el sentimiento de orientación vital en tres personas que han recibido diagnóstico de enfermedad mental" (Soru y Duero, 2011, p. 59). Se aborda el estudio a partir del sentimiento existencial o de orientación vital, que es una forma primaria de las experiencias humanas; son vivencias que influyen o determinan el significado que damos a nuestra existencia en el mundo.

6 El nombre de la investigación es "Sentimiento vital, vivencias corporales y estructuración del campo de la conciencia: una aproximación fenomenológico-narrativa”. 


\section{La orientación vital}

Se trataría de una disposición anímica general, de una modalidad de estructuración consciente, pero también de un conjunto de experiencias corporales primarias (como la vivencia de orientación en el tiempo y el espacio) que pueden ser más o menos difusas, y que se configuran - de forma unitaria- un modo de estar y proyectarnos con respecto a nuestro entorno y a los otros (Soru y Duero, 2011, p. 60).

Para Ratcliffe (citado por Soru y Duero 2011), "el sentimiento de orientación vital se encuentra anclado o encarnado en nuestras experiencias corporales" (s.p.). En este tipo de trastornos, como el mencionado en el estudio, se altera y perturba la orientación vital, esto es lo que sucede con algunas enfermedades mentales. Este tipo de afecciones traen como consecuencia experiencias o vivencias con cambios en la percepción y en la conciencia del propio cuerpo.

Otra investigación de corte sociológico y que presenta la visión de experiencia como vivencia es la realizada por Ferrante y Ferreira (2010), "El habitus de la discapacidad: la experiencia corporal de la dominación en un contexto económico periférico". Los autores proponen el estudio de las vivencias de personas con discapacidad a partir de un modelo que tome en cuenta las categorías de habitus, trayectoria social y violencia simbólica. La tesis que sustentan es que son diferentes las experiencias vividas por personas con discapacidad de zonas marginales en Argentina, con privaciones de distinto orden, pero en especial económico, con discapacitados de países del primer mundo, quienes tienen otras garantías para convivir con su problemática. Desde este tipo de estudio, la experiencia es asumida como vivencia, como formas interiores de accionar frente a acontecimientos particulares, que están relacionados con la propia corporalidad. En este sentido:

Tener una discapacidad supone la experiencia de un "cuerpo alienado", pero dicha experiencia variará de acuerdo a la posición ocupada en el espacio social. Ahora bien, ese cuerpo dista mucho de ser, simplemente, un substrato fisiológico neutro, un depositario o continente "natural" de la experiencia; muy al contrario, se trata de "un cuerpo para el otro" que es un producto social [...]. (Bourdieu, 1988, p. 204; citado por Ferrante y Ferreira, 2010, p. 87).
Desde este tipo de estudios sociológicos, las vivencias están influenciadas a partir de determinadas condiciones sociales, en donde el medio y el contexto son factores determinantes en la formación y estructuración del cuerpo.

Dentro de la misma línea de estas investigaciones, pero en este caso utilizando el relato (entrevista abierta) se cita la investigación de Ediht Flórez y Lucy María Reidl (2007) de la Universidad Nacional Autónoma de México, llamada "Corporalidad y uso de drogas: Estudio de caso de la experiencia subjetiva del cuerpo". El estudio tiene la particularidad de utilizar el relato como estrategia metodológica, que busca interpretar las experiencias corporales a partir de ciertas condiciones particulares de las personas objetos de investigación. En este caso, la narración autobiográfica corresponde a un consumidor de drogas, quien relata sus vivencias y aspectos relevantes en su vida que fueron construyendo sus formas de asumir y vivir su cuerpo. Como lo señalan las autoras, bajo una práctica como es el uso de drogas, se cambian las formas de percepción del propio cuerpo en relación con el tiempo y el espacio. Se podría decir que los sentidos perciben de una manera distinta cuando una persona está bajo los efectos de la droga. Por ejemplo, el sujeto que relata sus experiencia con respecto al consumo de drogas, comenta: "La experiencia fue mucho muy rara, asombrosa, incluso, me dio mucho miedo porque estaba viviendo algo nunca vivido, algo extraordinario para mis sentidos [...] ya después de mi primera experiencia lo volví a repetir buscando esas sensaciones tan extraordinaria" (Flórez y Reidl, 2007, p. 246).

Desde esta condición especial, la experiencia corporal es asumida como un "puro sentir", porque las sensaciones que la persona consumidora percibe son gratificantes, pero distorsionan la realidad. Al respecto, Bernard señala:

La experiencia corporal depende de la manera en que cada uno aprehende, siente y gobierna su propio cuerpo; pero también es el resultado de valores culturales y prácticas sociales que moldean las formas de sentirlo, conservarlo, experienciarlo y presentarlo en la escena social. (Citado por Flórez y Reidl, 2007, p. 241).

Con el mismo enfoque existen un número significativo de investigaciones, en donde contar o relatar las vivencias es el método común: 
Algunos estudios recientes han reportado la riqueza y la utilidad de los relatos de vida para investigar la experiencia subjetiva implicada en procesos corporales tales como: prácticas culturales de modificación corporal (Albin,2002); narrativas sobre la experiencia de la menstruación (Koutroulis, 2001); la experiencia corporal del sufrimiento y la enfermedad (Skultans, 2000); la experiencia de heridas en la columna vertebral de deportistas (Smith \& Sparkes, 2002); los significados de la salud y la discapacidad en mujeres con discapacidades (Tighe, 2001); la experiencia corporal post parto (Upton \& Han, 2003) y experiencias de adicción al alcohol (Zakrzewslo \& Mark, 2004); mostrando que el relato con el que la persona da cuenta de su experiencia, Constituye una vía privilegiada para acceder al terreno de la subjetividad. (Flórez y Reidl, 2007, p. 243).

Como vemos, las investigaciones descritas y las citadas en el párrafo anterior refieren a las vivencias que tiene determinado tipo de población con unas condiciones particulares de vida y para la cual dentro de sus propósitos está el de analizar las formas de construcción corporal, a partir del sentido y significado que cada persona da a sus experiencias y aprendizajes. En su mayoría, las investigaciones señaladas se refieren a situaciones particulares de adversidad, patologías o discapacidades y por eso las disciplinas que más se interesan por este tipo de condiciones son la psicología, la sociología y la medicina. Una característica en los estudios citados es que existe una circunstancia externa que altera la manera de percibir el propio cuerpo y que distorsiona las formas de conciencia corporal. Para el propósito de esta investigación sobre la experiencia corporal, se puede señalar que los estudios revisados narran vivencias que tienen determinado tipo de personas, con alteraciones en las estructuras biológicas, producto de las condiciones mencionadas (drogadicción, discapacidad, problemas mentales entre otros). Por eso, en estos casos, se debe hablar más que de experiencia de relatos de vivencias.

Con respecto a la búsqueda particular en la relación educación física-experiencia corporal no se encontraron estudios en este campo, los más cercanos son los ejemplos citados en los anteriores párrafos, sobre temas como experiencias de discapacitados o con deportistas en situaciones de adversidad. También, desde el análisis del estado del arte sobre la experiencia corporal, algunos de los estudios más pertinentes son los de la educación corporal, cuyo campo conceptual, sin ser específico de la educación física, puede ser horizonte para esta área. En este enfoque existen otras investigaciones que, como la de Flórez y Reidl, tienen una comprensión del cuerpo desde una visión fenomenológica y son abordadas a partir de las experiencias en la educación corporal.

Dentro de ellas hacemos referencia a la investigación de corte biográfico-narrativa de Gloria María Castañeda Clavijo (2009), quien da cuenta de la experiencia vivida a partir de la narrativa de tres bailarines, quienes realizan prácticas corporales dancísticas con regularidad. En la investigación es importante rescatar el valor "que el sujeto de la experiencia hace de sí mismo, en ese proceso de constitución de subjetividad-identidad, mismidad, individuación" (Castañeda, 2009, p. 6). El estudio devela la importancia de la reflexión de nuestras vivencias o de las prácticas corporales que dan significado a nuestra existencia. En este sentido, en la investigación referenciada se hace una crítica a los estudios de la educación física centrados en la "política del cuerpo" que, como lo plantea Bárcena y otros (2003):

Se refiere al abordaje de cuerpo-objeto que permanentemente es sometido a la dominación, control o fabricación; un territorio de experimentación, sea a través de prácticas de régimen disciplinario que el sujeto se impone a sí mismo o que proviene del exterior. (Citados en Castañeda, 2009, p. 6).

Además, la autora señala la poca reflexión que hace la educación física de las prácticas artísticas, que se queda solo en la acción y no lleva la práctica misma a una reflexión personal que la convertiría en una experiencia significativa. En la investigación se alcanza a inferir una semejanza en los conceptos de experiencia y vivencia, a partir de la referencia que la autora hace de Ortega y Gasset (1913), quien le dio sentido al término vivencia en la traducción de la palabra alemana erlebnis que quiere decir 'la experiencia vivida'. En cuanto a la experiencia, la investigadora se basó en la comprensión que hace Larrosa (citado por Castañeda, 2009), quien la define como "lo que nos pasa, nos acontece, o nos llega" (p. 12).

En el estudio, la autora cita a Taylor (1999) para hablar del sujeto de la experiencia, quien lo comprende como un ser que lucha por tener identidad, entendida esta como la conciencia de tener un lugar en el mundo, tener un punto de vista propio, tener una posibilidad 
de hablar por sí mismo y ser un "interlocutor". En este caso, si se hace referencia al sujeto de la experiencia, hablamos de que la experiencia acontece en el sujeto, en su corporeidad, que permite esa trascendencia, esa reflexión y esa comprensión de las prácticas, más allá de acciones repetitivas carentes de significado, alejadas de la propia subjetivad, o por condiciones particulares, como las señaladas en las investigaciones en donde las personas involucradas en el estudio relatan sus vivencias. Es por ello que en esta investigación el enfoque biográfico-narrativo es una forma de traspasar, de resignificar una práctica corporal, para darle sentido de existencia, es decir, una experiencia que acontece en lo corporal.

En la misma línea de la anterior investigación, se hace referencia a dos estudios: el primero realizado por la doctora Luz Elena Gallo (2011) con un enfoque teóricodocumental, que se desarrolla a partir de la educación corporal bajo la figura del acontecimiento. Dentro de la metodología, como lo señala la autora, retoma conceptos de Gilles Deleuze y Fernando Bárcena, para lograr conectar algunas concepciones de dichos autores e imbricarlas dentro de la educación corporal. Pero la pertinencia de esta investigación con respecto a la experiencia corporal está justo en la apropiación de la investigadora del concepto de acontecimiento, ya que logra darle un nuevo territorio en la educación corporal y, en ese sentido, devela el acontecimiento "como un estallido de sentidos, algo (nos) pasa, algo hace experiencia en nosotros y nos sorprende, nos orienta hacía algo nuevo porque no nos deja igual que antes" (Gallo, 2011, p. 505). Como se habla de la educación corporal y si, como lo señala la autora, "el acontecimiento constituye la experiencia. Lo que nos pasa en el cuerpo nos permite hacer experiencia educativa" (Gallo, 2011, p. 509), a partir de la cita anterior, la reflexión que se debe hacer es cómo desde la educación podemos privilegiar experiencias significativas.

Luz Elena Gallo aborda este interrogante a partir de lo que ella llama la "intensidad de la membrana", pero, para elaborar el concepto de membrana retoma una cita de Deleuze (1994, p. 19), este autor señaló:

Sólo las membranas ponen en contacto el interior y el exterior, lo profundo y lo alto, y gracias a esa superficie topológica de contacto podemos decir que el acontecimiento no ocupa la superficie, sino que aparece en ella, la energía superficial no está localizada en la superficie, sino ligada a su formación y reformación. (Gallo, 2011, p. 508).
Desde esta perspectiva, señala la autora que hay experiencia en la educación a partir de la intensidad, en donde la membrana forma la diferencia.

Se puede señalar, a partir de interpretar lo que plantea esta investigación, que el acontecimiento es experiencia que sucede en el sujeto, que se hace visible en su corporeidad. De ahí que la educación deba comprender el cuerpo como unidad, en donde convergen todas las dimensiones humanas, y que el aprendizaje significativo tiene que tener presente las sensaciones, las sensibilidades y los sentimientos que tiene cada persona, de esta manera se viven experiencias que tienen significado y afectan la existencia y las formas de convivencia.

Un segundo estudio que busca darle sentido a la educación corporal es el de Gómez, S. N. y Molina, N. (2011). Se basa en las hermenéuticas del sí mismo y asume la fenomenología hermenéutica y la antropología pedagógica como directriz epistémica de la educación corporal. Desde este enfoque metodológico se da sentido a la narrativa como un eje fundamental de la educación corporal que acerca el cuerpo-sujeto a la experiencia que logramos a partir del relato de vida. En el texto referenciado, las autoras retoman la interpretación que sobre la experiencia hace Larrosa (Gómez y Molina, 2011, p. 540 s.s.). Al respecto, un primer aporte es el que nos propone alejar la experiencia como concepto y debemos comprenderla a partir del sujeto. El proceso inicia con un acontecimiento, que se convierte en una experiencia significativa cuando hay una reflexión o tiene sentido y significado en la persona que lo vive. Larrosa, citado por Gómez y Molina (2011), nos aclara esta transformación:

La experiencia, como la posibilidad de que algo nos pase o nos acontezca o nos llegue, requiere un gesto de interrupción, un gesto que es casi imposible en los tiempos que corren: requiere pararse a pensar, pararse a mirar, pararse a escuchar, pensar más despacio, mirar más despacio y escuchar más despacio, parase a sentir, sentir más despacio, demorarse en los detalles, suspender la opinión suspender el juicio, suspender la voluntad, suspender el automatismo de la acción, cultivar la atención y la delicadeza, abrir los ojos y los oídos, charlar sobre lo que nos pasa, aprender la lentitud, escuchar a los demás, cultivar el arte del encuentro, callar mucho, tener paciencia, darse tiempo y espacio. (Larrosa, citado por Gómez y Molina, 2011, p. 541). 
Una vez hecha la reflexión sobre la comprensión del sentido que se debe dar al acontecimiento para relacionarlo con una experiencia de vida, se finaliza la descripción de este estudio planteando que el acto narrativo permite el acontecimiento y la experiencia. Desde este análisis, hay una relación con la investigación de Castañeda, quien también le da valor al relato narrativo.

Al relacionar los tres estudios descritos en los anteriores párrafos, vemos que lo que los particulariza es que al argumentar la re-significación de la educación corporal desde la narrativa, el acontecimiento o las hermenéuticas del sí mismo, se hace referencia a la experiencia corporal. De ahí, podemos concluir que la educación corporal favorece o se sustenta desde la experiencia vivida que se construye desde la interioridad, en la corporeidad.

Como cierre, se evidencia en el sucinto recorrido por los estudios de la experiencia corporal que se han realizado, que se puede entender, por un lado, la experiencia como acciones y vivencias que acontecen en situaciones de adversidad, en algunos casos reflejan problemáticas sociales y en otros nos muestran las dificultades que se tienen para superar ciertas adversidades. Como ya se señaló, estas investigaciones son de carácter sociológico, psicológico y médico y desde estos estudios debemos hablar de vivencias, más que experiencias. Por otro lado, encontramos otros estudios que evidencian una relación concreta entre la experiencia corporal y la educación corporal:

Una Educación Corporal permite explorar la corporeidad como otro lenguaje de la educación en tanto el ser humano es presencia corporal en el mundo. El cuerpo es construcción simbólica y esto puede ser interpretado desde los proceso de subjetivación que dan lugar a diferentes formas de expresión de las subjetividades. (Gallo, 2010, p. 307).

\section{CONCLUSIONES}

En conclusión, en esta revisión de algunos de los estudios sobre la experiencia corporal se puede señalar, que en las primeras investigaciones revisadas, más relacionadas con la historicidad de la experiencia, no se encuentra una diferenciación concreta entre el término experiencia y vivencia. Frente a sus diferencias, Andrés Díaz Velasco señala: "He aquí la relación experienciavivencia: la 'experiencia' no será la misma 'vivencia'; su diferencia consiste en que no todo lo vivido es una expe- riencia, aunque ésta se dé sólo y únicamente durante la otra" (Díaz, 2007, p. 89). Sin pretender reducir los dos términos o de desconocer la interpretación realizada por innumerables filósofos, para la investigación que nos ocupa y a partir del análisis de los estudios revisados, se habla de experiencia corporal como potencializadora de la corporeidad, en plenitud existencial y en donde emergen las dimensiones del ser humano. De allí que, en el caso de algunas de las investigaciones sobre la educación corporal, las prácticas tienen valor y significado para quien las realiza. Por el contrario, en las tres primeras investigaciones referenciadas, hablamos de situaciones que le suceden a personas con algunas condiciones especiales, allí se puede decir que se relatan las vivencias que tienen las personas objetos de investigación, con el propósito de servir de referencia para otras personas, en el sentido coloquial de que se aprende de las experiencias de otros. Luz Elena Gallo (2011) con la significación que hace de la experiencia nos deja ver otra de las diferencias existentes con lo que significa vivencia: "La experiencia es lo que nos pasa y que al pasarnos nos confiere sentido, la experiencia no es lo que te sucede, sino lo que haces con lo que te sucede" (p. 296). La vivencia es lo que nos sucede, y eso es lo que acontece en las primeras investigaciones revisadas, cuyo propósito es el análisis de las vivencias que le acontecen a los individuos objetos de la investigación. Allí se menciona el término experiencia corporal, pero desde la comprensión de la experiencia como vivencia.

A partir de la revisión de los estudios sobre la experiencia corporal, otra de las conclusiones con sentido educativo es que este campo no ha sido de interés para la educación física, por eso los hallazgos entre educación y experiencia corporal estuvieron centrados en la educación corporal. En estas investigaciones, algo característico es qué al significar la corporeidad, hacen referencia a la experiencia corporal como parte de lo esencial en la educación. "Una educación corporal va hacía la producción de sentido sobre el cuerpo-vivido y las experiencias vividas dejan ver cómo la propia persona experimenta su corporalidad" (Gallo, 2010, p. 315). Desde el planteamiento propuesto por la autora, se comprende que la corporeidad es abordada a partir de la concepción fenomenológica ${ }^{7}$. Desde allí la edu-

7 Autores como Merleau-Ponty, Huserl y Marcel entre otros centraron parte de sus reflexiones en torno a la fenomenología del cuerpo; al respecto Luz Elena Gallo (2006, p. 47) nos 
cación se instaura en lo corporal que entiende al ser humano como una unidad indivisible y se distancia de la dualidad antropológica y en especial desde el cartesianismo que lo asume como res extensa, materialidad, organicidad, es decir, en una postura biológica. Con el término corporal:

No se está haciendo alusión a las cosas materiales o extensivas ni a las propiedades dependientes de la naturaleza física del cuerpo, sino que lo corporal, puede ver la naturaleza del hombre como organismo y, a la vez, como cuerpo sensitivo y animado. El ser corporal es entonces indiviso. (Gallo, 2010, p. 302).

En este sentido, para la investigación que nos ocupa, la experiencia corporal se instaura en la corporalidad, en donde están presentes las dimensiones del ser humano y se diferencia de lo que se ha planteado como vivencia.

\section{REFERENCIAS}

Amengual, G. (2006). El concepto de la experiencia de Kant a Hegel (en línea). Recuperado de http://www.redalyc. org/articulo.oa?id=28811907001>ISSN1666-485X

Castañeda, G. (2009). Vivencia de prácticas corporales artísticas: allegar-se al cuerpo vivido desde las expresiones dancísticas (Trabajo investigativo para optar al título de magíster en Motricidad y Desarrollo humano). Universidad de Antioquia, Medellín.

Díaz, A. (2007). ¿Qué nos insinúa la experiencia corporal? Revista Lúdica Pedagógica, 2(12), 86-97.

dice que "la fenomenología del cuerpo abre un nuevo horizonte para comprender la dimensión corpórea de la existencia humana y ofrece una nueva visión filosófica del cuerpo, en tanto que el cuerpo no es sólo una realidad observable como objeto, sino que es una dimensión del propio ser".
Ferrante, C., y Ferreira M. (2010). El habitus de la discapacidad: la experiencia corporal de la dominación en un contexto económico periférico. Política $y$ Sociedad, 47(1), 85-104.

Flórez, E., y Reidl, L. (2007). Corporalidad y uso de drogas: estudio de caso de la experiencia subjetiva del cuerpo. Revista Interamericana de Psicología/ Interamerican Journal of Psychology, 41(2), 241-250.

Gallo, L. (2010). Los discursos de la educación física contemporánea. Bogotá: Kinesis.

Gallo, L. (2011). La educación corporal bajo la figura del acontecimiento. Revista Educación Física y Deporte, $30(2), 505-513$.

Gómez, S., y Molina, N. (2011). Las hermenéuticas del sí mismo: Un acercamiento comprensivo a las dimensiones de la educación corporal. Revista Educación Física y Deporte, 30(2), 539-549.

Gama, L. (2002). Una tensa cercanía: Hegel y Gadamer y el concepto de experiencia. Revista Ideas y Valores, (120), 41-78.

Hegel, G. (1995). Fenomenología del Espíritu. México: Fondo de Cultura Económica.

López Ibor, J. (s.f.). Charla sin título. Recuperado de: www. rampra.org/pag/pag/PDF/Conferencia 280411.pdf

Planella, J. (2006). Cuerpo, cultura y educación. Bilbao: Desclée De Brouwer.

Soru, M., y Duero, D. (2011). Experiencias corporales y sentimiento de orientación vital: una aproximación fenomenológica y narrativa. Revista Pensando Psicología, 7(13), 59-74. 